\title{
Evaluation of the BD PHOENIX Automated Microbiology System for Detection of Methicillin Resistance in Coagulase-Negative Staphylococci
}

\author{
Matthias A. Horstkotte, ${ }^{1 *}$ Johannes K.-M. Knobloch, ${ }^{1}$ Holger Rohde, ${ }^{1}$ Sabine Dobinsky, ${ }^{1}$ and \\ Dietrich Mack ${ }^{1,2}$ \\ Institut für Infektionsmedizin, Zentrum für Klinisch-Theoretische Medizin, Universitätsklinikum Hamburg-Eppendorf, Hamburg, \\ Germany, ${ }^{1}$ and Chair of Medical Microbiology and Infectious Diseases, The Clinical School, University of Wales Swansea, \\ Swansea, United Kingdom²
}

Received 14 April 2004/Returned for modification 31 May 2004/Accepted 24 July 2004

\begin{abstract}
The new BD PHOENIX automated microbiology system (Becton Dickinson Diagnostic Systems, Sparks, Md.) is designed for automated rapid antimicrobial susceptibility testing and identification of clinically relevant bacteria. In our study, the accuracy and speed of the BD PHOENIX oxacillin MIC determination for detecting methicillin resistance was evaluated for 200 clinical isolates of coagulase-negative staphylococci (CoNS). Compared to $m e c A$ PCR, the BD PHOENIX system detected methicillin resistance with a sensitivity of $99.2 \%$. According to the actual NCCLS oxacillin MIC breakpoint of $\geq 0.5 \mu \mathrm{g} / \mathrm{ml}$, the specificity was only 64.9\%, attributable to false-positive results in 26 mec $A$-negative strains, including 16 non-Staphylococcus epidermidis strains. Alternative oxacillin breakpoints of $\geq 1, \geq 2$, and $\geq 4 \mu \mathrm{g} / \mathrm{ml}$ resulted in increased specificities of 83.8, 94.6, and $100 \%$ and high sensitivities of 99.2, 99.2, and 96.7\%, respectively. Similarly, NCCLS broth microdilution oxacillin MICs exhibited a sensitivity of $100 \%$ but a low degree of specificity. However, the previous oxacillin MIC breakpoint of $\geq 4 \mu \mathrm{g} / \mathrm{ml}$ performed with a sensitivity of $98.4 \%$ and a specificity of $98.7 \%$. BD PHOENIX oxacillin MIC results were available after $9 \mathrm{~h}$ for $40.5 \%$ of the examined CoNS strains and were completed after $17 \mathrm{~h}$. Our results revealed the high reliability of the BD PHOENIX system as a phenotypic method for detection of resistance to oxacillin in mecA-positive CoNS. However, for the improvement of specificity, reevaluation of the optimal oxacillin MIC breakpoint for CoNS appears to be necessary.
\end{abstract}

Coagulase-negative staphylococci (CoNS) are major nosocomial pathogens ranking today among the five most frequently isolated organisms in nosocomial sepsis and infections related to foreign biomaterials, like intravascular and peritoneal dialysis catheters, cerebrospinal fluid shunts, prosthetic heart valves, and prosthetic joints, vascular grafts, cardiac pacemakers, and intraocular lenses (20, 26, 27, 35, 41, 43).

Frequent multiple antibiotic resistance, and especially the high prevalence of clinical CoNS exhibiting methicillin resistance, has turned treatment of CoNS infection into a therapeutic challenge $(2,10)$. Methicillin resistance is mediated by an additional low-affinity penicillin binding protein 2a (PBP2a) encoded by the mec $A$ gene (4).

For critically ill patients, vancomycin is the empirical therapy until methicillin resistance is ruled out. However, antistaphylococcal penicillins are the drugs of choice because of unexcelled activity and low toxicity and in order to reduce the possibility of the emergence of vancomycin-resistant CoNS, Staphylococcus aureus, and enterococci (6, 7, 31, 36, 37, 45). Reliable and rapid detection of methicillin resistance is thus indispensable for such "curtailed" antibiotic therapy of patients with CoNS infections. Detection of $m e c A$ by PCR is very sensitive and is considered the reference method (33). Alter-

\footnotetext{
* Corresponding author. Mailing address: Institut für Infektionsmedizin, Universitätsklinikum Hamburg-Eppendorf, Martinistrasse 52, D-20246 Hamburg, Germany. Phone: 49 40/42803-3147. Fax: 49 40/42803-4881. E-mail: horstkotte@uke.uni-hamburg.de.
}

natively, detection of $\mathrm{PBP} 2 \mathrm{a}$ as a marker of methicillin resistance has shown promising results with CoNS and is therefore also recommended $(1,15-17,25,33,44,48)$. In contrast, phenotypic detection of methicillin resistance in CoNS is difficult due to the heterogeneous expression of mec $A(6,30,40,47)$. Commonly used tests rely on modified culture conditions to enhance the expression of resistance and take up to $48 \mathrm{~h}$ for reliable results $(30,40,47)$.

Rapid availability of antimicrobial susceptibility data is likely to result in curtailed antibiotic therapy $(9,42)$, and lower mortality rates have been reported (9). Some automated systems for susceptibility testing offer the advantage of rapid reporting and improved detection of unusual resistance phenotypes, which is likely to significantly improve intra- and interlaboratory reproducibility. Additionally, financial benefits may result from reduced laboratory costs, fewer diagnostic tests performed, and decreased length of stay for patients in an intensive care unit $(3,9,12)$.

In our study, the accuracy and speed of the BD PHOENIX automated microbiology system (Becton Dickinson Diagnostic Systems, Sparks, Md.) for detecting methicillin resistance in CoNS were evaluated by comparing the results of mec $A$ PCR and oxacillin MIC determination by broth microdilution to the results of the BD PHOENIX oxacillin MIC determination for 200 clinical CoNS isolates.

(Part of this work will appear in the doctoral thesis of M. A. Horstkotte, Universitätsklinikum Hamburg-Eppendorf, Hamburg, Germany.) 


\section{MATERIALS AND METHODS}

Bacterial isolates. The CoNS strains in the present study $(n=200)$ comprise 14 different species (139 S. epidermidis strains, $16 \mathrm{~S}$. haemolyticus strains, $10 \mathrm{~S}$. hominis strains, 9 S. saprophyticus strains, 6 S. capitis strains, 4 S. lugdunensis strains, 4 S. warneri strains, 4 S. xylosus strains, 2 S. schleiferi strains, 2 S. cohni strains, $1 S$. chromogenes strain, $1 \mathrm{~S}$. simulans strain, $1 \mathrm{~S}$. arlettae strain, and $1 \mathrm{~S}$. kloosii strain). All of the strains belong to a collection of clinical CoNS isolated in 1997 and 1998 at the University Hospital Hamburg-Eppendorf. Species identification was performed by using Gram stain morphology, the catalase test, the clumping factor test, and the ID 32 Staph system (bioMérieux, Marcy l' Etoile, France). The strains were characterized for the presence of PBP2a using a commercially available latex agglutination test (Denka Seiken Co., Niigata, Japan) (15) and have been used for evaluation of the new automated VITEK2 system (14). The strains were kept at $-80^{\circ} \mathrm{C}$ in microbank tubes (Pro Lab Diagnostics, Richmond Hill, Ontario, Canada). S. aureus ATCC 29213 (mecA negative) and $S$. aureus ATCC 43300 (mecA positive) were used for quality control.

PCR for $\boldsymbol{m e c} \boldsymbol{A}$. PCRs were performed as described previously $(15,27)$

BD PHOENIX antimicrobial susceptibility testing. Methicillin resistance was detected by oxacillin MIC determination (range, $\leq 0.25$ to $\geq 8 \mu \mathrm{g} / \mathrm{ml}$ ) included in the PMIC/ID-6 test panel for gram-positive bacteria (reference no. 448513; Becton Dickinson) of the BD PHOENIX system according to the manufacturer's instructions. Briefly, each isolate was subcultured twice on Columbia agar (Difco, Becton Dickinson) containing 5\% sheep blood for $24 \mathrm{~h}$ at $37^{\circ} \mathrm{C}$ before being tested. The BD PHOENIX identification (ID) broth was inoculated with bacterial colonies of the respective isolate adjusted to a $0.5 \mathrm{McFarland}$ standard using a CrystalSpec Nephelometer (Becton Dickinson Diagnostic Systems). An aliquot of $25 \mu \mathrm{l}$ of the ID suspension was subsequently inoculated into the antimicrobial susceptibility test broth (Becton Dickinson), and 1 drop of the redox indicator resazurin was added. The self-inoculating combination panels were filled, sealed, and loaded into the BD PHOENIX system, where kinetic measurements of both colorimetric and fluorescent signals were collected every $20 \mathrm{~min}$. The results for the BD PHOENIX oxacillin MIC determination were recorded after every hour up to $9 \mathrm{~h}$ for each strain. Strains for which the oxacillin MICs were 0.5 to $2 \mu \mathrm{g} / \mathrm{ml}$ or with divergent oxacillin MIC results compared to the PCR for mecA were retested.

Broth microdilution. Each isolate was subcultured twice on Columbia agar containing $5 \%$ sheep blood at $37^{\circ} \mathrm{C}$ before being tested. Oxacillin MIC results were determined according to the guidelines of the NCCLS (33) with an inoculum of $5 \times 10^{5} \mathrm{CFU} / \mathrm{ml}$ in cation-adjusted Mueller-Hinton broth (Difco) supplemented with $2 \% \mathrm{NaCl}$. The polystyrol microtiter plates with U-shaped wells (Greiner, Kremsmünster, Austria) were incubated at $35^{\circ} \mathrm{C}$ and read after $24 \mathrm{~h}$.

\section{RESULTS}

mec $A$ was detected in 124 of $200(62 \%)$ CoNS strains, with 99 of $140(70.7 \%)$ mec $A$-positive $S$. epidermidis strains and 25 of $60(41.7 \%)$ mec $A$-positive non-S. epidermidis strains.

A BD PHOENIX oxacillin MIC of $\geq 8 \mu \mathrm{g} / \mathrm{ml}$ was reported for 115 of 123 mec $A$-positive strains. The BD PHOENIX oxacillin MICs for four $m e c A$-positive strains were $4 \mu \mathrm{g} / \mathrm{ml}$, whereas those for two mec $A$-positive $S$. epidermidis strains and one $m e c A$-positive $S$. saprophyticus strain were $2 \mu \mathrm{g} / \mathrm{ml}$. The oxacillin MIC of only one mecA-positive $S$. epidermidis strain was $\leq 0.25 \mu \mathrm{g} / \mathrm{ml}$ (Tables 1 and 2 ).

The BD PHOENIX system detected an oxacillin MIC of $\leq 0.25 \mu \mathrm{g} / \mathrm{ml}$ for 48 of $74 \mathrm{mec} A$-negative CoNS but oxacillin MICs of $0.5,1$, and $2 \mu \mathrm{g} / \mathrm{ml}$, respectively, for 14,8 , and 4 $m e c A$-negative strains. These higher oxacillin MICs were observed for 10 S. epidermidis strains, 6 S. saprophyticus strains, 4 S. lugdunensis strains, $1 \mathrm{~S}$. xylosus strains, $1 \mathrm{~S}$. capitis strain, $1 \mathrm{~S}$. haemolyticus strain, $1 S$. arlettae strain, $1 S$. kloosii strains, and $1 S$. cohnii strain (Tables 1, 2, 3, and 4).

The 29 strains for which there were BD PHOENIX oxacillin MICs ranging from 0.5 to $2 \mu \mathrm{g} / \mathrm{ml}$ and the $1 \mathrm{mec} A$-positive $S$. epidermidis strain for which there was a BD PHOENIX oxacil-
TABLE 1. BD PHOENIX oxacillin MICs compared to mecA PCR on the basis of different oxacillin MIC breakpoints ${ }^{a}$

\begin{tabular}{|c|c|c|c|c|}
\hline \multirow{2}{*}{$\begin{array}{c}\text { Oxacillin MIC } \\
\text { breakpoint }(\mu \mathrm{g} / \mathrm{ml}) \text { and } \\
\text { strain type }\end{array}$} & \multicolumn{2}{|c|}{$\begin{array}{l}\text { No. of CoNS strains } \\
\text { classified as: }\end{array}$} & \multirow{2}{*}{$\begin{array}{l}\text { Sensitivity } \\
(\%)\end{array}$} & \multirow{2}{*}{$\begin{array}{l}\text { Specificity } \\
(\%)\end{array}$} \\
\hline & Resistant & Susceptible & & \\
\hline \multicolumn{5}{|l|}{$\geq 0.5$} \\
\hline$m e c A$-positive & 122 & 1 & 99.2 & 64.9 \\
\hline $\begin{aligned} & m e c A \text {-negative } \\
\geq 1 & \end{aligned}$ & 26 & 48 & & \\
\hline$m e c A$-positive & 122 & 1 & 99.2 & 83.8 \\
\hline $\begin{array}{l}\operatorname{mec} A \text {-negative } \\
\geq 2\end{array}$ & 12 & 62 & & \\
\hline$m e c A$-positive & 122 & 1 & 99.2 & 94.6 \\
\hline$m e c A$-negative & 4 & 70 & & \\
\hline$\geq 4$ & & & & \\
\hline mec $A$-positive & 119 & 4 & 96.7 & 100 \\
\hline mec $A$-negative & 0 & 74 & & \\
\hline
\end{tabular}

${ }^{a}$ BD PHOENIX oxacillin MICs were not reported initially for 3 of 200 CoNS strains due to insufficient growth of the strains in the test panels. These three strains were not included this analysis.

lin MIC of $\leq 0.25 \mu \mathrm{g} / \mathrm{ml}$ were retested (Table 2). In the majority of strains, the oxacillin MIC results were within \pm 1 doubling dilution (Table 2).

For three strains, a mecA-negative $S$. epidermidis strain, a $m e c A$-negative $S$. cohnii strain, and a mecA-positive $S$. epidermidis strain, no BD PHOENIX oxacillin MIC was reported initially due to insufficient growth, but on retesting, the BD PHOENIX oxacillin MICs for the mecA-negative and the $m e c A$-positive $S$. epidermidis strains were 1 and $4 \mu \mathrm{g} / \mathrm{ml}$, respectively. For the mecA-negative $S$. cohnii strain, no BD PHOENIX result was obtained on retesting.

According to the actual resistant NCCLS oxacillin MIC breakpoint of $\geq 0.5 \mu \mathrm{g} / \mathrm{ml}$, the BD PHOENIX system detected methicillin resistance in CoNS with a sensitivity of $99.2 \%$ and a specificity of $64.9 \%$. Interestingly, the previous NCCLS oxacillin MIC breakpoint of $\geq 4 \mu \mathrm{g} / \mathrm{ml}$ would have displayed an excellent specificity of $100 \%$ and a high sensitivity of $96.7 \%$. Alternative oxacillin MIC breakpoints of $\geq 1$ and $\geq 2 \mu \mathrm{g} / \mathrm{ml}$ both had a sensitivity of $99.2 \%$ with specificities of 83.8 and $94.6 \%$, respectively (Table 2 ).

Broth microdilution testing among the $124 \mathrm{mec} A$-positive CoNS strains revealed oxacillin MICs of $\geq 8 \mu \mathrm{g} / \mathrm{ml}$ for 114 strains, $4 \mu \mathrm{g} / \mathrm{ml}$ for 8 strains, and 2 and $0.5 \mu \mathrm{g} / \mathrm{ml}$, respectively, for one $S$. epidermidis and one $S$. saprophyticus strain (Tables 2 , 3 , and 4). This resulted in a sensitivity of $100 \%$ for the actual resistant NCCLS oxacillin MIC breakpoint of $\geq 0.5 \mu \mathrm{g} / \mathrm{ml}$. However, the specificity reached only $13 \%$, with an oxacillin MIC of $\leq 0.25 \mu \mathrm{g} / \mathrm{ml}$ for 10 of $76 \mathrm{mec} A$-negative strains, whereas the oxacillin MICs for 27, 25, and 13 strains were 0.5 , 1 , and $2 \mu \mathrm{g} / \mathrm{ml}$, respectively. The broth microdilution oxacillin MIC for only one mec $A$-negative $S$. kloosii strain was $4 \mu \mathrm{g} / \mathrm{ml}$ (Tables 3 and 4). In contrast, the previous NCCLS oxacillin MIC breakpoint of $\geq 4 \mu \mathrm{g} / \mathrm{ml}$ performed with an improved specificity of $98.7 \%$ and a sensitivity of $98.4 \%$. Alternative oxacillin MIC breakpoints of $\geq 1$ or $\geq 2 \mu \mathrm{g} / \mathrm{ml}$ displayed a sensitivity of $99.2 \%$ and specificities of 48.7 and $81.6 \%$, respectively.

Comparison of BD PHOENIX oxacillin MICs and broth microdilution oxacillin MICs revealed that the MICs were 
TABLE 2. CoNS strains for which BD PHOENIX oxacillin MIC is 0.5 to $2 \mu \mathrm{g} / \mathrm{ml}$ or is discrepant compared to $m e c A$ PCR

\begin{tabular}{|c|c|c|c|c|c|}
\hline \multirow{3}{*}{ Strain no. } & \multirow{3}{*}{ Species } & \multicolumn{3}{|c|}{ Oxacillin MIC $(\mu \mathrm{g} / \mathrm{ml})$} & \multirow{3}{*}{$m e c A$ PCR } \\
\hline & & \multicolumn{2}{|c|}{ BD PHOENIX } & \multirow{2}{*}{$\begin{array}{l}\text { Broth } \\
\text { microdilution }\end{array}$} & \\
\hline & & Initially & On repeat testing & & \\
\hline 2 & S. epidermidis & 0.5 & $\leq 0.25$ & 0.25 & Negative \\
\hline 12 & S. epidermidis & 0.5 & $\leq 0.25$ & 0.5 & Negative \\
\hline 15 & S. epidermidis & 0.5 & No growth & 2 & Negative \\
\hline 174 & S. epidermidis & 0.5 & $\leq 0.25$ & 0.5 & Negative \\
\hline 199 & S. haemolyticus & 0.5 & 0.5 & 0.125 & Negative \\
\hline 64 & S. capitis & 0.5 & 0.5 & 2 & Negative \\
\hline 8 & S. lugdunensis & 0.5 & 0.5 & 2 & Negative \\
\hline 89 & S. lugdunensis & 0.5 & $\leq 0.25$ & 0.5 & Negative \\
\hline 175 & S. lugdunensis & 0.5 & 0.5 & 0.5 & Negative \\
\hline 7 & S. saprophyticus & 0.5 & 0.5 & 1 & Negative \\
\hline 9 & S. saprophyticus & 0.5 & 0.5 & 1 & Negative \\
\hline 48 & S. saprophyticus & 0.5 & 0.5 & 1 & Negative \\
\hline 177 & S. saprophyticus & 0.5 & 0.5 & 1 & Negative \\
\hline 95 & S. xylosus & 0.5 & 0.5 & 1 & Negative \\
\hline 1 & S. epidermidis & 1 & 1 & 0.25 & Negative \\
\hline 55 & S. epidermidis & 1 & $\leq 0.25$ & 0.25 & Negative \\
\hline 207 & S. epidermidis & 1 & 0.5 & 1 & Negative \\
\hline 173 & S. arlettae & 1 & 1 & 2 & Negative \\
\hline 90 & S. kloosii & 1 & 1 & 4 & Negative \\
\hline 16 & S. lugdunensis & 1 & 0.5 & 2 & Negative \\
\hline 3 & S. saprophyticus & 1 & 1 & 2 & Negative \\
\hline 4 & S. saprophyticus & 1 & 1 & 0.5 & Negative \\
\hline 50 & S. epidermidis & 2 & 0.5 & 1 & Negative \\
\hline 52 & S. epidermidis & 2 & $\leq 0.25$ & 0.5 & Negative \\
\hline 99 & S. epidermidis & 2 & $\leq 0.25$ & 0.5 & Negative \\
\hline 60 & S. cohnii & 2 & 2 & 2 & Negative \\
\hline 102 & S. epidermidis & $\leq 0.25$ & $\leq 0.25$ & 8 & Positive \\
\hline 73 & S. epidermidis & 2 & $\geq 8$ & 4 & Positive \\
\hline 204 & S. epidermidis & 2 & 1 & 2 & Positive \\
\hline 96 & S. saprophyticus & 2 & $\geq 8$ & 0.5 & Positive \\
\hline
\end{tabular}

within \pm 1 doubling dilution (essential agreement) for the majority of the examined strains (Table 3 and 4 ).

Analysis of the time to results of the BD PHOENIX system revealed that oxacillin MIC results were available after 8 and $9 \mathrm{~h}$ in 18.5 and $40.5 \%$ of the strains, respectively (Table 5). After 6 or $7 \mathrm{~h}, m e c A$-positive strains predominated, but after $8 \mathrm{~h}$, the proportions of $m e c A$-positive CoNS detected as resistant $(19.4 \%)$ and $m e c A$-negative CoNS reported as susceptible $(17.1 \%)$ were about equal (Table 5). However, after 9 h, mec $A$ negative CoNS reported as susceptible outweighed mec $A$-pos-

TABLE 3. One-to-one comparison of NCCLS broth microdilution oxacillin MIC results with BD PHOENIX oxacillin MIC results in $m e c A$-positive CoNS strains ${ }^{a}$

\begin{tabular}{ccccccr}
\hline $\begin{array}{c}\text { BD PHOENIX } \\
\text { oxacillin MIC } \\
(\mu \mathrm{g} / \mathrm{ml})\end{array}$ & \multicolumn{5}{c}{ No. of strains at NCCLS broth microdilution oxacillin } \\
& $\leq 0.25$ & 0.5 & 1 & 2 & 4 & $\geq 8$ \\
\hline & & & & & 6 & 109 \\
$\geq 8$ & & & & & 1 & 3 \\
4 & & 1 & & 1 & 1 & \\
2 & & & & & & \\
1 & & & & & & 1 \\
0.5 & & & & & & 1 \\
$\leq 0.25$ & & &
\end{tabular}

${ }^{a}$ BD PHOENIX oxacillin MICs were not reported initially for 3 of 200 CoNS strains due to insufficient growth of the strains in the test panels. These three strains were not included this analysis. itive CoNS reported as resistant, with 47.4 versus $36.3 \%$ (Table 5). BD PHOENIX oxacillin MIC testing was completed after 17 h (Table 5).

\section{DISCUSSION}

The BD PHOENIX system provides automated antimicrobial susceptibility testing and identification of clinically relevant bacteria and has been evaluated for the identification of Burkholderia cepacia complex isolates and the detection of

TABLE 4. One-to-one comparison of NCCLS broth microdilution oxacillin MIC results with BD PHOENIX oxacillin MIC results in $m e c A$-negative CoNS strains ${ }^{a}$

\begin{tabular}{ccrrrrr}
\hline $\begin{array}{c}\text { BD PHOENIX } \\
\text { oxacillin MIC } \\
(\mu \mathrm{g} / \mathrm{ml})\end{array}$ & \multicolumn{6}{c}{ No. of strains at NCCLS broth microdilution oxacillin } \\
\cline { 2 - 7 } & $\leq 0.25$ & 0.5 & 1 & 2 & 4 & $\geq 8$ \\
\hline$\geq 8$ & & & & & & \\
4 & & 2 & 1 & 1 & & \\
2 & 2 & 1 & 1 & 3 & 1 & \\
1 & 2 & 4 & 5 & 3 & & \\
0.5 & 6 & 19 & 17 & 6 & & \\
$\leq 0.25$ & & & & & & \\
\hline
\end{tabular}

${ }^{a}$ BD PHOENIX oxacillin MICs were not reported initially for 3 of 200 CoNS strains due to insufficient growth of the strains in the test panels. These three strains were not included this analysis. 
TABLE 5. Time course of BD PHOENIX oxacillin MIC results in $\mathrm{CoNS}^{a}$

\begin{tabular}{|c|c|c|c|c|c|c|}
\hline \multirow{2}{*}{ oNS } & \multicolumn{6}{|c|}{ No. $(\%)$ of strains with reported oxacillin MIC results at (h): } \\
\hline & 5 & 6 & 7 & 8 & 9 & 17 \\
\hline 11 & $1(0.5)$ & $8(4)$ & $21(10.5)$ & $37(18.5)$ & $81(40.5)$ & 197( \\
\hline mec $A \mathrm{p}$ & $1(0.8)$ & $8(6.5)$ & $18(14.5)$ & $24(19.4)$ & $45(36.3)$ & 123 \\
\hline$m e c A$ negative & $0(0)$ & $0(0)$ & $3(3.9)$ & $13(17.1)$ & $36(47.4)$ & $74(97.4)$ \\
\hline
\end{tabular}

${ }^{a}$ BD PHOENIX oxacillin MICs were not reported initially for 3 of 200 CoNS strains due to insufficient growth of the strains in the test panels. These three strains were not included this analysis.

extended-spectrum beta-lactamases in Escherichia coli and Klebsiella species $(5,22,38)$. Recently, the BD PHOENIX system has been tested with a variety of gram-positive bacteria. There was good agreement of oxacillin MIC results for 161 CoNS strains between the BD PHOENIX system and broth microdilution as the reference method (11). However, detailed information about discrepant strains was lacking and $m e c A$ PCR was performed only for strains already categorized as resistant by the oxacillin $\mathrm{MIC}$, rendering exact determination of sensitivity and specificity difficult. The time until detection of methicillin resistance was not addressed.

In our study, the new BD PHOENIX system proved to be a highly reliable phenotypic method for detecting $m e c A$-positive CoNS strains as resistant to oxacillin. Based on the current NCCLS oxacillin MIC breakpoint of $\geq 0.5 \mu \mathrm{g} / \mathrm{ml}$, the BD PHOENIX oxacillin MIC exhibited an excellent sensitivity of $99.2 \%$ but a rather low specificity of $64.9 \%$ (Table 1 ). These findings were supported by the broth microdilution results, which exhibited an excellent sensitivity of $100 \%$ but a specificity of $13 \%$. However, evaluation of the two oxacillin MIC determination methods with higher breakpoints would have shown a favorable performance.

In 1999, the NCCLS lowered the oxacillin MIC breakpoint for CoNS from $\geq 4$ to $\geq 0.5 \mu \mathrm{g} / \mathrm{ml}(33,40)$. Subsequently, several investigators found high sensitivity of the new breakpoint for S. epidermidis, S. hominis, and S. haemolyticus but described reduced specificity for $S$. saprophyticus, $S$. cohnii, $S$. xylosus, $S$. warneri, and $S$. lugdunensis strains $(13,19,25,34,40)$. Concordantly, in our study, six $S$. saprophyticus strains, four $S$. lugdunensis strains, one $S$. xylosus strain, one $S$. cohnii strain, one $S$. capitis strain, one $S$. arlettae strain, and one $S$. kloosii strain were mecA negative, but the BD PHOENIX oxacillin MICs for the strains ranged from 0.5 to $2 \mu \mathrm{g} / \mathrm{ml}$. The classification of these 15 isolates was confirmed by broth microdilution results (Table 2).

Similarly, decreased specificity of the actual breakpoint was described for the Vitek automated system (bioMérieux), predominantly due to mec $A$-negative $S$. saprophyticus and $S$. lugdunensis and to a minor extent to $S$. cohnii, S. sciuri, and $S$. capitis strains $(18,25,29,34,46)$. The new VITEK 2 system (bioMérieux) showed high sensitivity $(14,21,23,24)$, and concordance between mecA PCR and VITEK 2 oxacillin MICs was observed for almost all $S$. epidermidis, $S$. haemolyticus, and $S$. hominis strains $(14,23)$. However, the specificities varied considerably, between 97 and $80 \%$, due to false-positive results, especially for mecA-negative $S$. saprophyticus, $S$. cohnii, and $S$. lugdunensis strains $(14,23)$.

It is notable that in our study BD PHOENIX oxacillin MICs of $0.5,1$, and $2 \mu \mathrm{g} / \mathrm{ml}$ were reported for four, three, and three $m e c A$-negative $S$. epidermidis strains, respectively, as well as for one mecA-negative $S$. haemolyticus strain (Table 2). Broth microdilution would have classified 4 of these 11 isolates as susceptible (Table 2). Essential agreement between the oxacillin MICs in the two methods was recorded for four of the remaining seven strains, which all had to be classified as resistant (Table 2). The 10 (7.2\%) mecA-negative $S$. epidermidis strains for which the oxacillin MICs were $\geq 0.5 \mu \mathrm{g} / \mathrm{ml}$, out of a total of 139 S. epidermidis strains, represent a proportion similar to that in another study, which found 2 of 37 (5.4\%) S. epidermidis strains for which the oxacillin MICs were $\geq 0.5 \mu \mathrm{g} / \mathrm{ml}(30)$.

For epidemiological reasons, study populations vary in their species compositions and strains differ in their phenotypic expression of methicillin resistance. Consequently, a range of oxacillin MIC breakpoints for resistance have been proposed. Until 1999, the NCCLS recommended an oxacillin MIC breakpoint for resistance in all staphylococci of $\geq 4 \mu \mathrm{g} / \mathrm{ml}$, but $\geq 2$ (47) or $\geq 1(8,30) \mu \mathrm{g} / \mathrm{ml}$ was advocated as more appropriate for CoNS. An even lower oxacillin MIC breakpoint of $\geq 0.5$ $\mu \mathrm{g} / \mathrm{ml}$, suggested by two other investigators (28, 40), was adopted for CoNS by the NCCLS in 1999.

The inoculum size is crucial for oxacillin MIC determination. Despite adherence to the NCCLS protocol, the inoculum was clearly below the target concentration of $5 \times 10^{5} \mathrm{CFU} / \mathrm{ml}$ for all CoNS species except $S$. lugdunensis in a large multicenter study $(33,40)$. Interestingly, the recorded mean numbers of CFU per milliliter were lower for $S$. epidermidis, $S$. hominis, and $S$. haemolyticus than for $S$. saprophyticus, $S$. capitis, and S. lugdunensis (40), which correlates with the higher oxacillin MICs described in several studies for the last three species despite their being $\operatorname{mec} A$ negative.

Subtle changes in the test conditions, e.g., the source of the Mueller-Hinton medium, have been reported to affect oxacillin MIC results for CoNS (40). The BD PHOENIX antimicrobial susceptibility test broth contains $0.01 \%$ Tween 80 , which may explain some discrepancies in oxacillin MIC results.

Mechanisms of oxacillin resistance independent of mec $A$ may be responsible for oxacillin MIC results of 0.5 to $2 \mu \mathrm{g} / \mathrm{ml}$ for $m e c A$-negative strains. Alterations in PBPs other than PBP2a have been described in S. haemolyticus and S. saprophyticus (39) and have been suggested as one explanation for this phenomenon (40). Alternatively, S. saprophyticus and $S$. lugdunensis, and probably certain other CoNS species, might inherently display a higher oxacillin MIC despite lacking mec $A$.

To improve the specific detection of methicillin resistance in CoNS, exclusion of S. saprophyticus and S. lugdunensis strains was recommended (25), and this proposal was adopted in part by the NCCLS, which no longer recommends routine oxacillin susceptibility testing of $S$. saprophyticus isolated from urine (33).

However, this issue cannot be fully resolved by the exclusion of $S$. saprophyticus and $S$. lugdunensis strains or by the recommendation of the NCCLS to additionally analyze CoNS strains other than $S$. epidermidis which cause severe infections for the presence of either PBP2a or mecA and to consider strains lacking these markers as susceptible. PCR for mec $A$ is both laborious and costly, whereas false-positive PBP2a latex agglutination tests have been observed for mec $A$-negative $S$. warneri, $S$. lugdunensis, $S$. simulans, and $S$. hominis strains but not $S$. 
epidermidis strains $(1,15,16,44,48)$. The question of whether penicillinase-resistant penicillins can eradicate severe infections due to $m e c A$-negative CoNS for which the oxacillin MICs are 0.5 to $2 \mu \mathrm{g} / \mathrm{ml}$ is unresolved. Clinical trials with strains of $S$. lugdunensis, which is now regarded as an important pathogen in native valve endocarditis (32), or S. saprophyticus causing complicated urinary tract infection are needed.

In our study, all CoNS strains for which the BD PHOENIX oxacillin MICs were 0.5 or $1 \mu \mathrm{g} / \mathrm{ml} \operatorname{did}$ not harbor $m e c A$, in contrast to three out of seven strains for which the oxacillin MIC was $2 \mu \mathrm{g} / \mathrm{ml}$. In another study, mec $A$ PCR results and the presence of PBP2a have been found to be identical in these CoNS strains (15). We conclude that CoNS strains for which the critical BD PHOENIX oxacillin MIC is $2 \mu \mathrm{g} / \mathrm{ml}$ should be examined for the presence of PBP2a or mecA. Further investigations are warranted to clarify whether differential, speciesspecific oxacillin MIC breakpoints of $\geq 2 \mu \mathrm{g} / \mathrm{ml}$ for $S$. saprophyticus and $S$. lugdunensis $(19,40)$ and $\geq 0.5 \mu \mathrm{g} / \mathrm{ml}$ for $S$. epidermidis and other CoNS species are more appropriate.

An optimized antibiotic regimen can result in lower mortality $(9,42)$. Therefore, antimicrobial test results should be accessible on the day of testing. Evaluation of the speed of the BD PHOENIX system for providing oxacillin MIC results revealed at least equivalence to those of other phenotypic methods. After $9 \mathrm{~h}$, results for $\sim 41 \%$ of the CoNS strains were reported, and oxacillin MIC testing was completed after $17 \mathrm{~h}$ (Table 5).

\section{ACKNOWLEDGMENTS}

We thank Rainer Laufs for his continuous support. Becton Dickinson (Heidelberg, Germany) kindly provided the BD PHOENIX automated microbiology system.

This work was supported in part by a grant from the Deutsche Forschungsgemeinschaft given to D.M.

\section{REFERENCES}

1. Andrews, J. M., F. J. Boswell, and R. Wise. 2000. Establishing MIC breakpoints for coagulase-negative staphylococci to oxacillin. J. Antimicrob. Chemother. 45:259-261.

2. Archer, G. L., and M. W. Climo. 1994. Antimicrobial susceptibility of coag ulase-negative staphylococci. Antimicrob. Agents Chemother. 38:2231-2237.

3. Barenfanger, J., C. Drake, and G. Kacich. 1999. Clinical and financial benefits of rapid bacterial identification and antimicrobial susceptibility testing. J. Clin. Microbiol. 37:1415-1418

4. Berger-Bächi, B. 1997. Resistance not mediated by $\beta$-lactamase (methicillin resistance), p. 158-174. In K. B. Crossley and G. L. Archer (ed.), The staphylococci in human disease. Churchill Livingston, New York, N.Y

5. Brisse, S., S. Stefani, J. Verhoef, A. van Belkum, P. Vandamme, and W. Goessens. 2002. Comparative evaluation of the BD PHOENIX and VITEK 2 automated instruments for identification of isolates of the Burkholderia cepacia complex. J. Clin. Microbiol. 40:1743-1748.

6. Chambers, H. F. 1997. Methicillin resistance in staphylococci: molecular and biochemical basis and clinical implications. Clin. Microbiol. Rev. 10:781-791.

7. Chang, S., D. M. Sievert, J. C. Hageman, M. L. Boulton, F. C. Tenover, F. P. Downes, S. Shah, J. T. Rudrik, G. R. Pupp, W. J. Brown, D. Cardo, and S. K. Fridkin. 2003. Infection with vancomycin-resistant Staphylococcus aureus containing the vanA resistance gene. N. Engl. J. Med. 348:1342-1347.

8. Cormican, M. G., W. W. Wilke, M. S. Barrett, M. A. Pfaller, and R. N. Jones. 1996. Phenotypic detection of $m e c A$-positive staphylococcal blood stream isolates: high accuracy of simple disk diffusion tests. Diagn. Microbiol. Infect. Dis. 25:107-112.

9. Doern, G. V., R. Vautour, M. Gaudet, and B. Levy. 1994. Clinical impact of rapid in vitro susceptibility testing and bacterial identification. J. Clin. Microbiol. 32:1757-1762.

10. Edmond, M. B., S. E. Wallace, D. K. McClish, M. A. Pfaller, R. N. Jones, and R. P. Wenzel. 1999. Nosocomial bloodstream infections in United States hospitals: a three-year analysis. Clin. Infect. Dis. 29:239-244.

11. Fahr, A. M., U. Eigner, M. Armbrust, A. Caganic, G. Dettori, C. Chezzi, L. Bertoncini, M. Benecchi, and M. G. Menozzi. 2003. Two-center collaborative evaluation of the performance of the BD PHOENIX automated microbiol- ogy system for identification and antimicrobial susceptibility testing of En terococcus spp. and Staphylococcus spp. J. Clin. Microbiol. 41:1135-1142.

12. Ferraro, M. J., and J. H. Jorgensen. 1999. Susceptibility testing instrumentation and computerized expert systems for data analysis and interpretation, p. 1593-1600. In P. R. Murray, E. J. Baron, M. A. Pfaller, F. C. Tenover, and R. H. Yolken (ed.), Manual of clinical microbiology. American Society for Microbiology, Washington, D.C.

13. Gradelski, E., L. Valera, L. Aleksunes, D. Bonner, and J. Fung-Tomc. 2001. Correlation between genotype and phenotypic categorization of staphylococci based on methicillin susceptibility and resistance. J. Clin. Microbiol. 39:2961-2963.

14. Horstkotte, M. A., J. K. M. Knobloch, H. Rohde, S. Dobinsky, and D. Mack. 2002. Rapid detection of methicillin resistance in coagulase-negative staphylococci with the VITEK 2 system. J. Clin. Microbiol. 40:3291-3295.

15. Horstkotte, M. A., J. K. M. Knobloch, H. Rohde, and D. Mack. 2001. Rapid detection of methicillin resistance in coagulase-negative staphylococci by a penicillin binding protein $2 \mathrm{a}$-specific latex agglutination test. J. Clin. Microbiol. 39:3700-3702.

16. Hussain, Z., L. Stoakes, S. Garrow, S. Longo, V. Fitzgerald, and R. Lannigan. 2000. Rapid detection of $m e c A$-positive and $m e c A$-negative coagulasenegative staphylococci by an anti-penicillin binding protein $2 \mathrm{a}$ slide latex agglutination test. J. Clin. Microbiol. 38:2051-2054.

17. Hussain, Z., L. Stoakes, M. A. John, S. Garrow, and V. Fitzgerald. 2002. Detection of methicillin resistance in primary blood culture isolates of coagulase-negative staphylococci by PCR, slide agglutination, disk diffusion, and a commercial method. J. Clin. Microbiol. 40:2251-2253.

18. Hussain, Z., L. Stoakes, R. Lannigan, S. Longo, and B. Nancekivell. 1998. Evaluation of screening and commercial methods for detection of methicillin resistance in coagulase-negative staphylococci. J. Clin. Microbiol. 36:273274.

19. Hussain, Z., L. Stoakes, V. Massey, D. Diagre, V. Fitzgerald, S. El Sayed, and R. Lannigan. 2000. Correlation of oxacillin MIC with $m e c A$ gene carriage in coagulase-negative staphylococci. J. Clin. Microbiol. 38:752-754.

20. Kloos, W. E. 1997. Taxonomy and systematics of staphylococci indigenous to humans, p. 113-137. In K. B. Crossley and G. L. Archer (ed.), The staphylococci in human disease. Churchill Livingston, New York, N.Y.

21. Leclercq, R., M. H. Nicolas-Chanoine, P. Nordmann, A. Philippon, P. Marchais, A. Buu-Ho, H. Chardon, H. Dabernat, F. Doucet-Populaire, C. Grasmick, P. Legrand, C. Muller-Serieys, J. Nguyen, M. C. Ploy, M. E. Reverdy, M. Weber, and R. J. Courcol. 2001. Multicenter evaluation of an automated system using selected bacteria that harbor challenging and clinically relevant mechanisms of resistance to antibiotics. Eur. J. Clin. Microbiol. Infect. Dis. 20:626-635.

22. Leverstein-van Hall, M. A., A. C. Fluit, A. Paauw, A. T. Box, S. Brisse, and J. Verhoef. 2002. Evaluation of the Etest ESBL and the BD Phoenix, VITEK 1, and VITEK 2 automated instruments for detection of extended-spectrum beta-lactamases in multiresistant Escherichia coli and Klebsiella spp. J. Clin. Microbiol. 40:3703-3711.

23. Ligozzi, M., C. Bernini, M. G. Bonora, M. De Fatima, J. Zuliani, and R. Fontana. 2002. Evaluation of the VITEK 2 system for identification and antimicrobial susceptibility testing of medically relevant gram-positive cocci. J. Clin. Microbiol. 40:1681-1686.

24. Livermore, D. M., M. Struelens, J. Amorim, F. Baquero, J. Bille, R. Canton, S. Henning, S. Gatermann, A. Marchese, H. Mittermayer, C. Nonhoff, K. J. Oakton, F. Praplan, H. Ramos, G. C. Schito, J. Van Eldere, J. Verhaegen, J. Verhoef, and M. R. Visser. 2002. Multicenter evaluation of the VITEK 2 Advanced Expert System for interpretive reading of antimicrobial resistance tests. J. Antimicrob. Chemother. 49:289-300.

25. Louie, L., A. Majury, J. Goodfellow, M. Louie, and A. E. Simor. 2001. Evaluation of a latex agglutination test (MRSA-Screen) for detection of oxacillin resistance in coagulase-negative staphylococci. J. Clin. Microbiol. 39:4149-4151.

26. Mack, D. 1999. Molecular mechanisms of Staphylococcus epidermidis biofilm formation. J. Hosp. Infect. 43(Suppl.):S113-S125.

27. Mack, D., K. Bartscht, S. Dobinsky, M. A. Horstkotte, K. Kiel, J. K. M. Knobloch, and P. Schäfer. 2000. Staphylococcal factors involved in adhesion and biofilm formation on biomaterials, p. 307-330. In Y. H. An and R. J. Friedman (ed.), Handbook for studying bacterial adhesion: principles, methods, and applications. Humana Press, Totowa, N.J.

28. Marshall, S. A., W. W. Wilke, M. A. Pfaller, and R. N. Jones. 1998. Staph ylococcus aureus and coagulase-negative staphylococci from blood stream infections: frequency of occurrence, antimicrobial susceptibility, and molecular $(m e c A)$ characterization of oxacillin resistance in the SCOPE program. Diagn. Microbiol. Infect. Dis. 30:205-214.

29. Martinez, F., L. J. Chandler, B. S. Reisner, and G. L. Woods. 2001. Evaluation of the Vitek card GPS105 and VTK-RO7.01 software for detection of oxacillin resistance in clinically relevant coagulase-negative staphylococci. J. Clin. Microbiol. 39:3733-3735.

30. McDonald, C. L., W. E. Maher, and R. J. Fass. 1995. Revised interpretation of oxacillin MICs for Staphylococcus epidermidis based on mecA detection. Antimicrob. Agents Chemother. 39:982-984. 
31. Murray, B. E. 2000. Vancomycin-resistant enterococcal infections. N. Engl J. Med. 342:710-721.

32. Mylonakis, E., and S. B. Calderwood. 2001. Infective endocarditis in adults. N. Engl. J. Med. 345:1318-1330.

33. National Committee for Clinical Laboratory Standards. 2003. Performance standards for antimicrobial susceptibility testing; 13th informational supplement. M100-S13. National Committee for Clinical Laboratory Standards, Wayne, Pa.

34. Ramotar, K., W. Woods, and B. Toye. 2001. Oxacillin susceptibility testing of Staphylococcus saprophyticus using disk diffusion, agar dilution, broth microdilution, and the Vitek GPS-105 card. Diagn. Microbiol. Infect. Dis. 40:203-205.

35. Rupp, M. E., and G. L. Archer. 1994. Coagulase-negative staphylococci: pathogens associated with medical progress. Clin. Infect. Dis. 19:231-243.

36. Small, P. M., and H. F. Chambers. 1990. Vancomycin for Staphylococcus aureus endocarditis in intravenous drug users. Antimicrob. Agents Chemother. 34:1227-1231.

37. Smith, T. L., M. L. Pearson, K. R. Wilcox, C. Cruz, M. V. Lancaster, B. Robinson-Dunn, F. C. Tenover, M. J. Zervos, J. D. Band, E. White, W. R Jarvis, et al. 1999. Emergence of vancomycin resistance in Staphylococcus aureus. N. Engl. J. Med. 340:493-501.

38. Stürenburg, E., I. Sobottka, H. H. Feucht, D. Mack, and R. Laufs. 2003 Comparison of BDPhoenix and VITEK2 automated antimicrobial susceptibility test systems for extended-spectrum beta-lactamase detection in Escherichia coli and Klebsiella species clinical isolates. Diagn. Microbiol. Infect. Dis. 45:29-34.

39. Suzuki, E., K. Hiramatsu, and T. Yokota. 1992. Survey of methicillin-resistant clinical strains of coagulase-negative staphylococci for mec $A$ gene distribution. Antimicrob. Agents Chemother. 36:429-434.

40. Tenover, F. C., R. N. Jones, J. M. Swenson, B. Zimmer, S. McAllister, and J. H. Jorgensen. 1999. Methods for improved detection of oxacillin resis- tance in coagulase-negative staphylococci: results of a multicenter study. J. Clin. Microbiol. 37:4051-4058.

41. Thylefors, J. D., S. Harbarth, and D. Pittet. 1998. Increasing bacteremia due to coagulase-negative staphylococci: fiction or reality? Infect. Control Hosp. Epidemiol. 19:581-589.

42. Trenholme, G. M., R. L. Kaplan, P. H. Karakusis, T. Stine, J. Fuhrer, W. Landau, and S. Levin. 1989. Clinical impact of rapid identification and susceptibility testing of bacterial blood culture isolates. J. Clin. Microbiol. 27:1342-1345.

43. U.S. Department of Health and Human Services. 2000. National Nosocomial Infections Surveillance (NNIS) system report, data summary from January 1992-April 2000, issued June 2000. Am. J. Infect. Control 28:429-448.

44. Udo, E. E., E. M. Mokadas, A. Al Haddad, B. Mathew, L. E. Jacob, and S. C Sanyal. 2000. Rapid detection of methicillin resistance in staphylococci using a slide latex agglutination kit. Int. J. Antimicrob. Agents 15:19-24.

45. Wilson, W. R., A. W. Karchmer, A. S. Dajani, K. A. Taubert, A. Bayer, D. Kaye, A. L. Bisno, P. Ferrieri, S. T. Shulman, D. T. Durack, et al. 1995 Antibiotic treatment of adults with infective endocarditis due to streptococci, enterococci, staphylococci, and HACEK microorganisms. JAMA 274:17061713.

46. Yamazumi, T., I. Furuta, D. J. Diekema, M. A. Pfaller, and R. N. Jones. 2001 Comparison of the Vitek gram-positive susceptibility 106 card, the MRSAScreen latex agglutination test, and $m e c A$ analysis for detecting oxacillin resistance in a geographically diverse collection of clinical isolates of coagulase-negative staphylococci. J. Clin. Microbiol. 39:3633-3636.

47. York, M. K., L. Gibbs, F. Chehab, and G. F. Brooks. 1996. Comparison of PCR detection of $m e c A$ with standard susceptibility testing methods to determine methicillin resistance in coagulase-negative staphylococci. J. Clin. Microbiol. 34:249-253.

48. Zbinden, R., M. Ritzler, E. Ritzler, and B. Berger-Bächi. 2001. Detection of penicillin-binding protein 2 a by rapid slide latex agglutination test in coagulase-negative staphylococci. J. Clin. Microbiol. 39:412. 